\title{
CAVITIES FOR JLAB'S 12 GEV UPGRADE*
}

\section{J. Sekutowicz, G. Ciovati, P. Kneisel, G. Wu, TJNAF, Newport News, 23606 VA, USA}

A. Brinkmann, DESY, 22603 Hamburg, FRG

W. Hartung, MSU, East Lansing, Michigan, USA

R. Parodi, INFN Genoa, 16146 Genoa, Italy

S. Zheng, Tsinghua University, 100084 Beijing, China

\section{Abstract}

The future $12 \mathrm{GeV}$ upgrade of CEBAF requires new cryomodules in both linacs to increase the energy gain per pass to $1090 \mathrm{MeV}$ [1]. Until recently, the design of new cryomodules, which should deliver on average operational voltage of $70 \mathrm{MV}$ each, was based on 7-cell superconducting cavities that are an extended version of the 5-cell structures currently used in the machine. The 5cell cavities were constructed 20 years ago at Cornell University (Original Cornell-shape) for the Cornell Electron Storage Ring (CESR). The geometry of these structures [2] met specifications at the time CESR was constructed but is not optimized for the future operation of CEBAF. Two improved cavity shapes have been proposed. This contribution presents the RF features of both new shapes and discusses advantages for the machine operation resulting from the improvement. In addition, we comment on the measurements on copper models of both new cavities and present results of the multipacting calculations.

\section{INTRODUCTION}

\section{High Gradient and Low Loss Shapes}

The High Gradient ( $\mathrm{HG}_{2}$ shape has been optimized to lower the ratio of $\mathrm{E}_{\text {peak }} / \mathrm{E}_{\text {acc }}$, keeping in mind that the limitation in the performance of superconducting cavities is in most cases field emission [3]. This phenomenon leads very often to electron showers captured by accelerating fields and/or to strong radiation increasing cryogenic loss and possibly causing a quench. By reducing this ratio one gains in the operational accelerating gradient even without any improvements in surface cleaning procedures.

The Low Loss (LL) shape has been optimized to increase both the characteristic impedance $((\mathrm{R} / \mathrm{Q}))$ and the geometry factor $(G)$ [4]. The improved parameters for this cavity result in less stored energy and less wall loss, at a given accelerating gradient, compared to the two other cavities. The reduced cryogenic load makes the nominal operation less expensive and opens the possibility to reach higher end energy of CEBAF within an available cryogenic budget.

\section{COMPARISON OF INNER CELLS}

In a multi-cell $\beta=1$ elliptical structure the RF properties of the accelerating mode are mainly determined by the geometry of the inner cells. The difference in the current and the proposed cells' shape can be seen in Fig. 1. Their parameters are listed in Table 1.

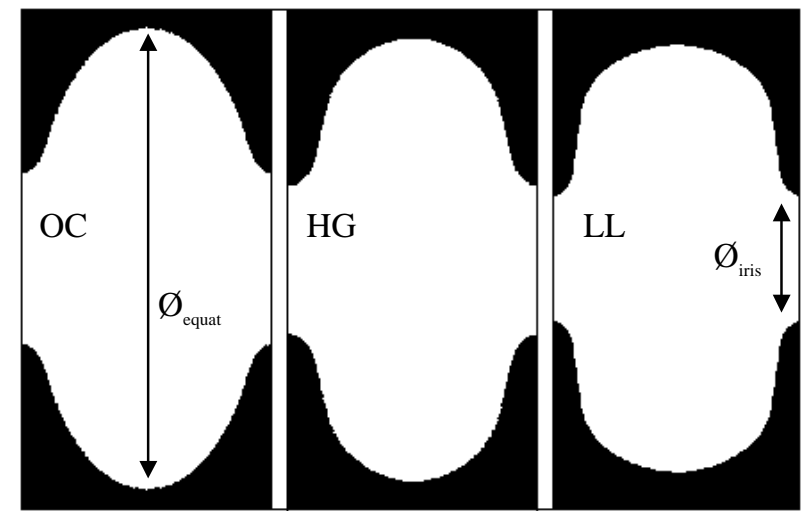

Figure 1: Geometry of three inner cells.

Table 1. Parameters of inner cells

\begin{tabular}{lrccc}
\hline \hline Parameter & & OC & HG & LL \\
\hline$\emptyset_{\text {equator }}$ & {$[\mathrm{mm}]$} & 187.0 & 180.5 & 174.0 \\
$\emptyset_{\text {iris }}$ & {$[\mathrm{mm}]$} & 70.0 & 61.4 & 53.0 \\
$\mathrm{k}_{\text {cc }}$ & {$[\%]$} & 3.29 & 1.72 & 1.49 \\
$\mathrm{E}_{\text {peak }} / \mathrm{E}_{\text {acc }}$ & {$[-]$} & 2.56 & 1.89 & 2.17 \\
$\mathrm{~B}_{\text {peak }} / \mathrm{E}_{\text {acc }}$ & {$[\mathrm{mT} /(\mathrm{MV} / \mathrm{m})]$} & 4.56 & 4.26 & 3.74 \\
$\mathrm{R} / \mathrm{Q}$ & {$[\Omega]$} & 96.5 & 111.9 & 128.8 \\
$\mathrm{G}$ & {$[\Omega]$} & 273.8 & 265.5 & 280.3 \\
$\mathrm{R} / \mathrm{Q} \cdot \mathrm{G}$ & {$[\Omega \cdot \Omega]$} & 26422 & 29709 & 36103 \\
\hline \hline
\end{tabular}

\section{Cell-to-cell coupling, $k_{c c}$}

The LL-shape has the smallest diameter of the equator and of the iris. The small aperture increases (R/Q) and $G$, but makes cell-to-cell coupling, $\mathrm{k}_{\mathrm{cc}}$, weaker. This increases the sensitivity of the field profile of the accelerating mode to frequency errors of individual cells. The measure of the sensitivity is the ratio: $\mathrm{N}^{2} /\left(\mathrm{k}_{\mathrm{cc}}\right)$, where $\mathrm{N}$ is the number of cells in a structure. This ratio, which one likes to keep low even if $\mathrm{k}_{\mathrm{cc}}$ is small, can be in a

\footnotetext{
* Work supported by the U.S.DOE under contract No. DE-AC05-84ER40150
} 
practical range by reducing the number of cells in a cavity. The upgrade cavities will be made of 7 cells. The ratio for the three upgrade shapes stays below the value for the 9-cell TESLA cavities $\left(\mathrm{k}_{\mathrm{cc}}=1.9 \%\right)$, of which almost 60 were manufactured up to now [5]. Their final field profile flatness, better than $95 \%$, was maintained without additional technical complications. The large iris of the OC cavity makes the coupling unnecessarily large causing other of its parameters to become unfavorable. The HG-shape is already more progressive here and closing the iris results in better (R/Q) and better $\mathrm{E}_{\text {peak }} / \mathrm{E}_{\text {acc }}$ of this shape.

$E_{\text {peak }} / E_{\text {acc }}$ ratio

Shape optimization with respect to this parameter is very important if cavities are operated at high gradients. However, when final preparation of the superconducting surface and the assembly in cryostat are properly done, the emission phenomena and the resulting radiation can be eliminated to a large degree. The design accelerating gradient for the upgrade has been set to $\mathrm{E}_{\mathrm{acc}} \approx 20 \mathrm{MV} / \mathrm{m}$ and $\mathrm{Q}_{\mathrm{o}}=8 \cdot 10^{9}$ at $2 \mathrm{~K}$. The nominal gradient needed to reach $12 \mathrm{GeV}$ of the end energy (with all cavities on) is $E_{n}=17.7 \mathrm{MV} / \mathrm{m}$. The possible operation of new cavities at higher accelerating field is limited by the cryogenic load budget. The maximum possible gradient is $18.3 \mathrm{MV} / \mathrm{m}$, 19.3 MV/m and 21.3 MV/m for OC-, HG- and LL-shape respectively. Thus the maximum possible $\mathrm{E}_{\text {peak }}$ is limited to: $47 \mathrm{MV} / \mathrm{m}$ for the OC-shape, $36 \mathrm{MV} / \mathrm{m}$ for the $\mathrm{HG}$ shape and $46 \mathrm{MV} / \mathrm{m}$ the for LL-shape. All shapes stay well below technically possible achievements, demonstrated for example, by many multi-cell TESLA cavities and many tests of single cell cavities worldwide.

$B_{\text {peak }} / E_{\text {acc }}$ ratio

Defects (e.g. residual Ta or embedded impurities) in the $\mathrm{Nb}$ surface can limit cavity performance and usually lead to quenches. It has been demonstrated that an additional chemical treatment or mechanical grinding which removes defects in the material can be a remedy to reach higher $\mathrm{E}_{\text {acc }}$. Local heating in the defect which eventually causes a quench, is proportional to its size, its surface resistance and the square of the local magnetic field. Therefore it is obvious, that for the same material quality and at a given gradient, cavities with lower magnetic field at the wall are favorable. The potential gain in $\mathrm{E}_{\text {acc }}$ offered by the LL-shape as compared to the OC-shape and to the HG-shape is $22 \%$ and $14 \%$ respectively. However for the limitation in gradient discussed above, $\mathrm{B}_{\text {peak }}$ of all shapes remains below $85 \mathrm{mT}$, a value routinely reached in tests of various $\mathrm{Nb}$ cavities.

\section{$(R / Q)$ and geometric factor $G$}

As already mentioned, (R/Q) and $\mathrm{G}$ determine the wall loss $\mathrm{P}_{\text {wall }}$. When the surface resistance $\left(\mathrm{R}_{\mathrm{S}}\right)$ and the gradient are given increasing the product $(\mathrm{R} / \mathrm{Q}) \cdot \mathrm{G}$ makes the wall loss lower:

$$
\mathrm{P}_{\text {wall }}=\mathrm{R}_{\mathrm{s}} \cdot\left(\mathrm{E}_{\text {acc }} \cdot \mathrm{L}_{\text {cell }}\right)^{2} /(\mathrm{G} \cdot(\mathrm{R} / \mathrm{Q}))
$$

where $\mathrm{L}_{\text {cell }}$ is the length of a cell. The LL-shape has the smallest wall loss. The loss of the OC-shape and of the HG-shape is higher by $35 \%$ and $21 \%$, respectively.

The second advantage of an increased (R/Q) is a lower value of the matched $\mathrm{Q}_{\mathrm{ext}}$ and thus a widening of the resonance width. This makes the cavity less sensitive to microphonics.

\section{7-CELL CAVITIES}

\section{Accelerating mode}

Table 2 shows (R/Q) values for all three 7-cell cavities and their parameters for the nominal operation. The value of the matched quality factor, $\mathrm{Q}_{\text {matched }}$, is computed for the total beam current of $460 \mu \mathrm{A}$ but without microphonics. The last row gives the optimized quality factor

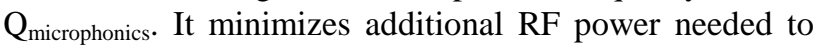
compensate for the maximum expected frequency mismatch of $25 \mathrm{~Hz}$ which may result from the microphonics and the resolution of a cold tuner [6].

Table 2. (R/Q) and parameters at $\mathrm{E}_{\mathrm{n}}=17.7 \mathrm{MV} / \mathrm{m}$.

\begin{tabular}{|c|c|c|c|c|}
\hline \multicolumn{2}{|c|}{ Parameters } & $\mathrm{OC}$ & $\mathrm{HG}$ & $\mathrm{LL}$ \\
\hline $\mathrm{R} / \mathrm{Q}$ & {$[\Omega]$} & 678 & 773 & 877 \\
\hline $\mathrm{Q}_{\mathrm{o}}$ & & $8 \cdot 10^{9}$ & $7.8 \cdot 10^{9}$ & $8.3 \cdot 10^{9}$ \\
\hline Dynamic RF- loss & at $2 \mathrm{~K}$ [W] & 28 & 25 & 21 \\
\hline $\mathrm{E}_{\text {peak }}$ & {$[\mathrm{MV} / \mathrm{m}]$} & 45 & 33 & 38 \\
\hline $\mathrm{B}_{\text {peak }}$ & {$[\mathrm{mT}]$} & 81 & 75 & 66 \\
\hline $\mathrm{Q}_{\text {matched }}$ & {$\left[10^{7}\right]$} & 4.0 & 3.5 & 3.1 \\
\hline $\mathrm{Q}_{\text {microphonics }}$ & {$\left[10^{7}\right]$} & 1.6 & 1.5 & 1.4 \\
\hline
\end{tabular}

The multipacting phenomenon can limit the performance of superconducting cavities. Both new shapes have been examined for this phenomenon with codes used at DESY, MSU and at INFN Genoa. All three codes proved that both shapes can be safely operated at gradients as high as $25 \mathrm{MV} / \mathrm{m}$ with no danger of multipacting. We will verify this result very soon with the first $\mathrm{Nb}$ prototypes of both new cavities.

\section{Damping of HOMs}

Dipole modes can degrade the quality of the CEBAF beam. Computed dipoles with high (R/Q) of both new cavities are listed in Table 3. The required suppression of these modes was computed with the help of the TDBBU code [7]. The results confirmed that the previous specification, $\mathrm{Q}_{\mathrm{ext}} \leq 1 \cdot 10^{6}$, as computed for the OC shape [8] holds for the new cavities also. The new cavities will have four HOM couplers, two at each beam tube (see Fig. 2 ). The couplers are based on the coaxial line technique and are similar to those used for the TTF cavities [9]. We tested this suppression scheme on copper models of new cavities. The results are shown in Fig. 3 
Table 3. Dipoles of new cavities .

\begin{tabular}{c|cc|cc}
\hline \hline & \multicolumn{2}{|c|}{ HG } & \multicolumn{2}{c}{ LL } \\
\hline No. & $\begin{array}{c}\mathrm{f} \\
{[\mathrm{MHz}]}\end{array}$ & $\begin{array}{c}\mathrm{R} / \mathrm{Q} \\
{\left[\Omega / \mathrm{cm}^{2}\right]}\end{array}$ & $\begin{array}{c}\mathrm{f} \\
{[\mathrm{MHz}]}\end{array}$ & $\begin{array}{c}\mathrm{R} / \mathrm{Q} \\
{\left[\Omega / \mathrm{cm}^{2}\right]}\end{array}$ \\
\hline 1 & 1980.27 & 15.9 & 1978.94 & 6.8 \\
2 & 2017.60 & 15.2 & 2013.55 & 10.1 \\
3 & 2060.48 & 3.0 & 2136.47 & 2.6 \\
4 & 2143.71 & 4.1 & 2170.61 & 7.2 \\
5 & 2150.87 & 7.5 & 2191.31 & 15.1 \\
6 & 2155.11 & 1.9 & 2207.37 & 8.2 \\
7 & 3004.67 & 18.9 & 2893.92 & 38.1 \\
8 & & & 2900.13 & 4.0 \\
9 & \multicolumn{4}{|l}{} \\
\hline \hline
\end{tabular}

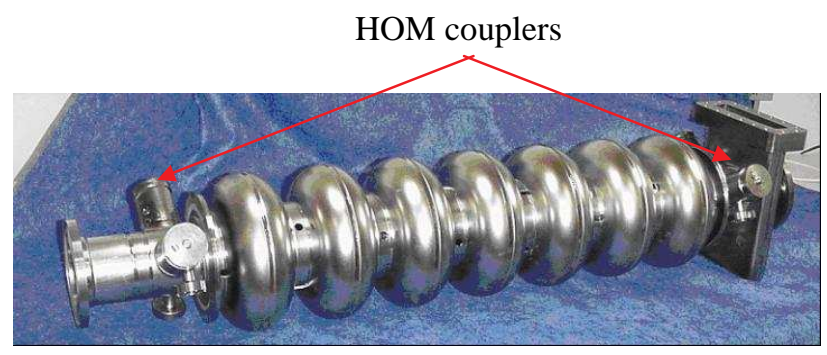

Figure 2: $\mathrm{Nb}$ prototype of $\mathrm{HG}$ cavity with four HOM couplers.

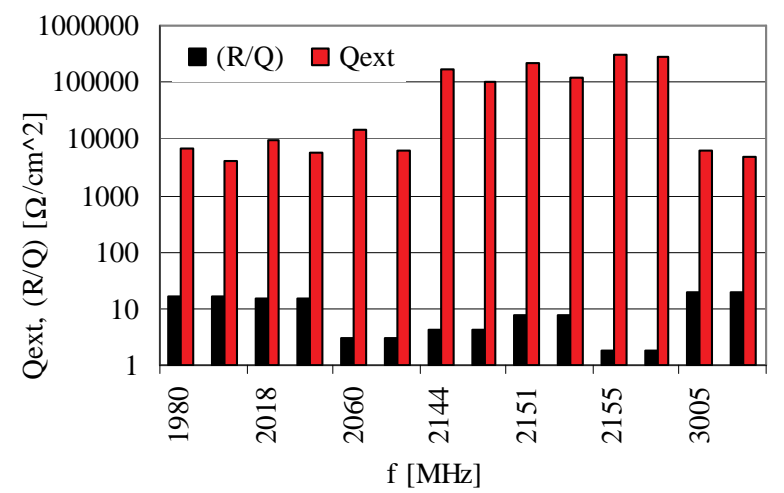

Figure 3: HG cavity. Measured damping of dipoles.

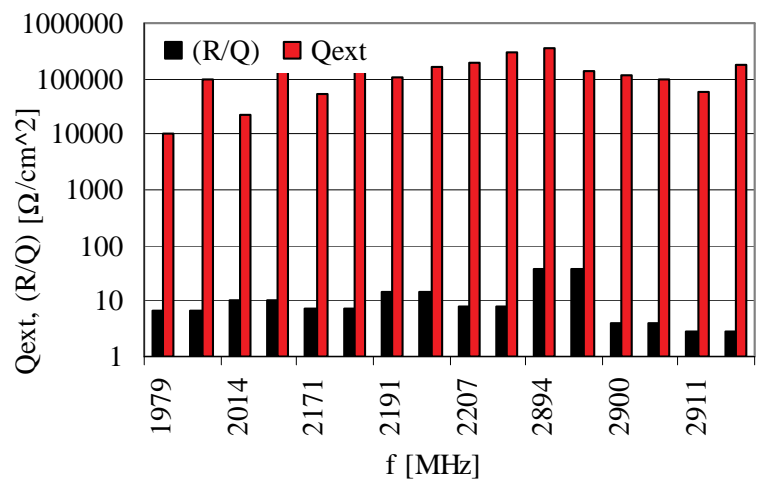

Figure 4: LL cavity. Measured damping of dipoles. and Fig. 4 for the HG- and the LL-cavity respectively. The LL cavity has weaker cell-to-cell coupling for the parasitic modes than the HG cavity. Their field profile are more sensitive to imperfection in shape and, in case of propagating modes, to the frequency of a neighboring cavity. We have investigated the damping of the propagating dipole No. 7 of LL cavity vs. various standing wave patterns in the interconnecting tube [10]. Except for the one case, when the frequency and the polarization of both cavities were exactly the same, we could still obtain enough damping of this mode. The unfavorable situation is very improbable and no special means have been undertaken to avoid this situation [11].

\section{SUMMARY}

Two new cavity designs with improved properties have been completed for the CEBAF upgrade. A prototype of each has been manufactured and will be tested in the near future. The design of new test cryomodule, housing four cavities of each type, is in progress [12]. We are planing to perform the beam test to verify RF properties of the proposed cavities in 2004.

\section{ACKNOWLEDGMENTS}

We would like to express our gratitude to L. Turlington, J. Brawley, R. Bundy, D. Forehand, S. Manning, B. Manus, S. Morgan, G. Slack and E. Daly for their help in manufacturing of the models and the first $\mathrm{Nb}$ prototypes. We also like to thank $\mathrm{H}$. Wang for his MAFIA calculation of dipole modes for the very first version of the cavities.

\section{REFERENCES}

[1] Interim Point Design for the CEBAF $12 \mathrm{GeV}$ Upgrade, CEBAF, May 1999.

[2] P. Kneisel et al. , " Performance of SC Storage Ring Cavities at $1500 \mathrm{MHz}$ ", IEEE Trans. On Magn., MAG-21 (2), 1985.

[3] G. Ciovati et al. , "An Improved Cavity Design For The CEBAF Upgrade", JLAB-TN 01-015, 2001.

[4] J. Sekutowicz et al., "Low Loss Cavity for the $12 \mathrm{GeV}$ CEBAF Upgrade“", JLAB TN-02-023, June 2002.

[5] E. Haebel et al., "Cavity Shape Optimization for a Superconducting Linear Collider", HEACC, Vol. 2, Hamburg, 1992.

[6] L. Harwood et al. , "CEBAF at 12 and $24 \mathrm{GeV}$ ", SRF Workshop, Tsukuba, September 2001.

[7] K. Beard, private communication

[8] B. C. Yunn, "Dipole HOM Damping Requirement of New 7-Cell Cavity for the $12 \mathrm{GeV}$ CEBAF Upgrade", JLAB-TN 01-028, June 2001.

[9] J. Sekutowicz, "Higher Order Mode Coupler for TESLA", SRF Works., Newport News, October 1993.

[10] G. Wu et al., "HOM Damping Measurements on HG and LL Copper Models", JLAB-TN 03-012, 2003.

[11] C. Reece, private communication.

[12] E. Daly et al., " Improved Prototype Cryomodule for the CEBAF $12 \mathrm{GeV}$ Upgrade", this Proceedings. 